\title{
ORDERED STRUCTURES IN A VARIABLE LENGTH A.C. TRAP
}

\author{
V.N. Gheorghe, L. Giurgiu, O. Stoican, D. Cacicovschi, R. Molnar \\ AND B. MihalCEA* \\ Institute of Atomic Physics, INFLPR, 76900 Bucharest, P.O.Box MG-6, Romania
}

(Received November 10, 1997)

A variable length linear trap operating between $20-1000 \mathrm{~Hz}$ under standard temperature and pressure conditions is reported. We simulated and observed various kinds of stable patterns: linear strings, planar zig-zag and volume structures. The interparticle distances depend on the trapped particle number and trap length. Our setup allows trapped microparticle diagnosis by various methods.

PACS numbers: $32.80 . P j$

\section{Introduction}

The appearance of stable and ordered patterns for different charged microparticles contained in a conventional Paul trap in vacuum, at hundreds of $\mathrm{Hz}$, was already reported [1]. Later, crystallization for atomic ions in traps has also been achieved [2].

A simple and low-cost trapping setup can help in drawing conclusions about charged particle evolution in an ion trap. The laws describing trapped microparticle motion in air are the same with those accounting for stored atomic ion motion in ultrahigh vacuum. Microparticle breaking via air friction is analogous with ion cooling via buffer gas molecules collisions. Experimental setups for microscopical particle storage in Paul traps operating at standard temperature and pressure (STP) and $50 \mathrm{~Hz}$ with didactical demonstrative purpose [3] or for mass and charge, vapour pressure, viscosity and surface tension measurement have been successfully used [4]. For other possible applications, working with a variable length linear trap at STP conditions for different drive frequencies may be important.

The paper is organized as follows. After introducing the equations of motion for the trapped microparticles (Sec. 2), we present our setup with its electrical supply and the results obtained (Sec. 3). Finally, some concluding remarks are made.

*e-mail: viorica@alpha1.infim.ro 


\section{Basics}

The equation of motion for a particle of mass $M$ and charge $Q$ within the trap is

$$
\boldsymbol{F}=Q \boldsymbol{E}-K \boldsymbol{r}+M \boldsymbol{g}+Q \boldsymbol{E}_{\mathrm{dc}}
$$

where $r=(x, y, z)$ is the particle vector position and $K(K>0)$ is the coefficient describing the aerodynamical drag force. $\boldsymbol{E}$ is the trap electric field, $M \boldsymbol{g}$ is the gravitational force and $\boldsymbol{E}_{\mathrm{dc}}=V_{1} /\left(2 r_{0}\right)$ is the electric field produced by applying a static potential difference $V_{1}$ between the upper and lower rods separated at $2 r_{0}$, in order to shift the particles to the trap centre. Introducing the $\tau=\Omega t / 2$ and $\Lambda=K /(M \Omega)$ parameters, where $\Omega$ is the drive frequency, and defining $X=\mathrm{e}^{\Lambda \tau} x, Y=\mathrm{e}^{\Lambda \tau} y, Z=\mathrm{e}^{\Lambda \tau} z$, the Mathieu equations describing the trapping process are homogeneous on the $X$ and $Y$ axes, with the known solutions and stability domains. The $Z$-axis motion is described by an inhomogeneous Mathieu equation. In this case, stability is obtained for (a) $i \mu$ real; (b) $\mu$ real and $|\mu|<\Lambda$; (c) $\mu-i$ real and $|\mu-i|<\Lambda$. Hence, stability regions for solutions of the inhomogeneous equations of motion in presence of drag forces include not only the stability regions of the homogeneous equations but. also a part of the instability regions, being consequently greater.

Usually, trapped microparticle motion parametrical excitation is achieved by applying a low amplitude and variable frequency supplementary a.c. voltage in series with the $V_{\mathrm{ac}}$ voltage. When the supplementary a.c. field frequency is twice that of the secular motion, microparticles (located at the limit of the first Mathieu stability domain) resonantly absorb energy from the field, while their secular motion amplitude exponentially increases. If the trap contains a number of noninteracting particles, besides normal resonance at the secular motion frequency and parametrical resonance at the double secular frequency, other weak resonances were observed [5], as a consequence of the presence of coupled terms for various combinations of the motion frequencies.

When the coupling parameter between particles (the ratio between Coulombian and thermal energy) is greater than 168, solid effects occur in the stored particle system [1]. Because the interparticle distance is laiger for trapped microscopic particles than for trapped ions, it is much easier to observe such behaviour.

\section{Experimental arrangement and result}

\subsection{Experimental setup}

Our trap consists of four brass rods $a, b, d, \bar{d}$ equidistantly spaced on an approximately $1 \mathrm{~cm}$ radius (Fig. 1). The trap length is variable, because one of the endcaps $(\bar{c})$ is a piston cylinder sliding on the brass bars.

The trap is covered with a plastic transparent foil, which protects it against air streams. The foil is provided with a slit in order to enable microparticle introduction within the trap, by means of a syringe needle. Microparticles have been illuminated with an aim to visualize them, using either a low power halogen lamp $(20 \mathrm{~W})$ or a low power $\mathrm{He}-\mathrm{Ne}$ laser $(1 \mathrm{~mW})$ travelling along the trap axis. Both trap endcaps are pierced, thus allowing laser beam incidence and emergence. 


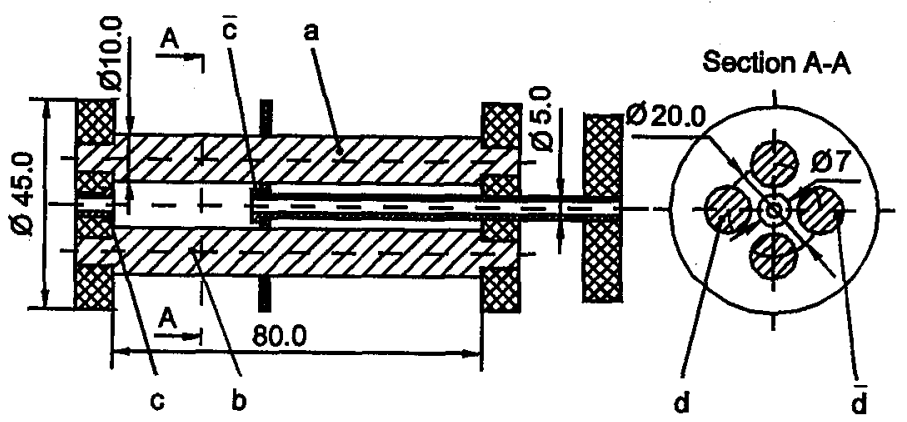

Fig. 1. Sketch of the trap geometry.

\subsection{Power supply}

A $V_{\text {ac }}(\Omega)$ variable voltage is applied between opposed pairs of rods (Fig. 2), in order to achieve trapping. A d.c.variable voltage $V_{1}(0-500 \mathrm{~V})$ vertically shifts the microparticle position. To avoid microparticle escape along the axis, two brass disks $c, \bar{c}$ with a $V_{2}$ d.c. bias $(0-500 \mathrm{~V})$ are provided. The $O 1$ oscillator delivers an a.c. voltage of $\Omega$ drive frequency and the $O 2$ oscillator delivers an a.c. voltage of $\omega$ frequency. Both voltage amplitudes are variable.

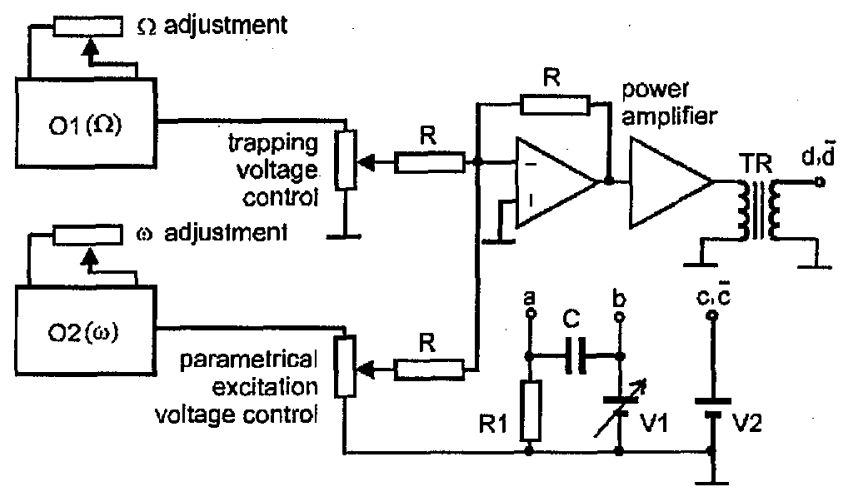

Fig. 2. Electrical supply scheme.

\subsection{Experimental results}

As microparticle specia we have-used SiC with homogeneous, well-defined dimensions rating between 50-1000 $\mu \mathrm{m}$. One up to thousands of microparticles can be stored along the trap axis. Depending on the trap length and $N$ trapped microparticle number we have obtained different $d$ interparticle distances (Fig. 3 ). 


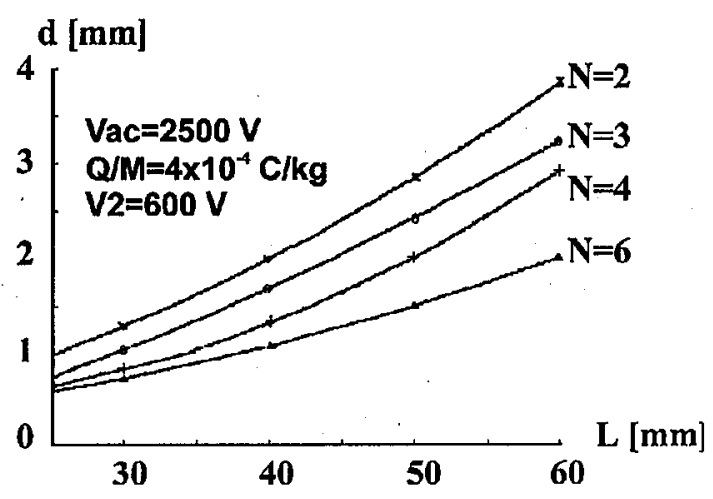

Fig. 3. Interparticle distance variation.

The $Q / M$ microparticle specific charge has been estimated from the $M g=$ $Q \boldsymbol{E}_{\mathrm{dc}}$ relation as about $10^{-4} \mathrm{C} / \mathrm{Kg}$ for $V_{1}=500 \mathrm{~V}$. The absolute electric charge has been evaluated from the electrostatical equilibrium conditions for microparticles stored on the trap axis, considering the $V_{2}$ produced electrical forces and interparticle Coulombian repulsion. For $V_{2}=500 \mathrm{~V}$ and $L=75 \mathrm{~mm}$, a $Q$ of about $10^{-11} \mathrm{C}$ results; from the specific charge value, the microparticle mass is evaluated. Knowing the $\mathrm{SiC}$ density value, we inferred the mean particle radius. The experimentally obtained values are consistent with the catalogue values.

Another diagnosis method used was parametrical excitation of the microparticle motion. The method implies the following. The signals delivered by the $O 1$ and $O 2$ oscillators (Fig. 2) are added and the resulting voltage is then fed into the $T R$ high voltage transformer primary. The $T R$ transformer secondary supplies both the trapping voltage of $\Omega / 2 \pi$ frequency and the parametrical excitation voltage of $\omega / 2 \pi$ frequency. When $\omega$ is twice the secular motion frequency, parametrical resonance occurs. Experimental measurements performed for $100 \mu \mathrm{m}$ diameter $\mathrm{SiC}$ microparticles, $\Omega / 2 \pi=100 \mathrm{~Hz}$ and $V_{\mathrm{ac}}=2.5 \mathrm{kV}$ have shown parametrical resonance at about $25 \mathrm{~Hz}$. Hence, microparticle specific charge was evaluated as $5 \times 10^{-4} \mathrm{C} / \mathrm{Kg}$.

\section{Conclusions}

We have achieved the trapping phenomenon for a variable length linear a.c. trap operating between $20 \mathrm{~Hz}-1 \mathrm{kHz}$. Our device allows microparticle diagnosis by various methods. We observed very stable different patterns similar to those appearing in high vacuum $[2,6]$.

\section{Acknowledgments}

The authors are very indebted to Prof. J. Dembczyński, Dr. E. Stachowska, Dr. D. Stefańska, Dr. M. Elantkowska and Dr. B. Arcimowicz for fruitful discussions on the subject. 


\section{References}

[1] H. Shelton, R.F. Wuerker, Bull. Am. Phys. Soc. 2, 375 (1957).

[2] H. Walther, Phys. Scr. T 51, 11 (1994).

[3] H. Winter, H.W. Ortjohann, Am. J. Phys. 59, 807 (1991).

[4] S. Arnold, L.M. Folan, A. Korn, J. Appl. Phys. 74, 4291 (1993).

[5] F. Vedel, M. Vedel, Phys. Rev. A 41, 5 (1990).

[6] M.G. Raisen, J.M. Gilligan, J.C. Bergquist, W.M. Itano, D.J. Wineland, J. Mod. Opt. 39, 233 (1992). 